\title{
RevM10 Gene
}

National Cancer Institute

\section{Source}

National Cancer Institute. RevM10 Gene. NCI Thesaurus. Code C18318.

RevM10 is a dominant-negative mutant of HIV-1 Rev gene, which encodes an RNAbinding protein involved in nuclear trafficking of unspliced viral mRNAs. The fact that Rev is essential for HIV-1 replication makes it an attractive targ et for antiviral approaches. Based on the notion of intracellular immunization, in vitro studies that introduce RevM10 gene into stem cells have demonstrated its ability to combat HIV-1 infection in human hematopoietic cells. 